\title{
The D-, A-, E- and T-optimal Values of a Second Order Rotatable Design in Four Dimension Constructed Using Balanced Incomplete Block Designs
}

\author{
Kabue Timothy Gichuki ${ }^{1}$, Koske Joseph ${ }^{2}$, Mutiso John ${ }^{2}$ \\ ${ }^{1}$ Department of Physical and Mathematical Sciences, School of pure and applied Sciences, Mount Kenya University, Thika, Kenya \\ ${ }^{2}$ Department of Statistics and Computer Science, School of Biological Sciences, Moi University Eldoret, Kenya
}

Email address:

kabuegichuki@yahoo.com (K. T. Gichuki), koske4@yahoo.co.uk (K. Joseph), johnkasome@yahoo.com (M. John)

\section{To cite this article:}

Kabue Timothy Gichuki, Koske Joseph, Mutiso John. The D-, A-, E- and T-optimal Values of a Second Order Rotatable Design in Four Dimension Constructed Using Balanced Incomplete Block Designs. American Journal of Applied Mathematics. Vol. 8, No. 3, 2020 , pp. 83-88. doi: $10.11648 /$ j.ajam.20200803.12

Received: March 5, 2020; Accepted: April 10, 2020; Published: May 14, 2020

\begin{abstract}
In response surface methodology, optimal designs are experimental designs generated based on a particular optimality criterion and are optimal only for a specific statistical model. Optimality criterion are single number criteria sometimes called alphabetical optimality criteria where each one intends to capture an aspect of the 'goodness' of a design. Most studies on optimization of process variables have concentrated on Central Composite Designs (CCD) yet second order rotatable deigns with any number of factors with reasonably small number of points constructed using properties of balanced incomplete block designs exist. A class of experimental designs that are optimal with respect to some statistical criterion are said to be Optimal designs. These designs allow parameter estimation with increased precision using fewer experimental runs, without bias and with minimum variance thus reducing time and costs of experimentation as opposed to non-optimal designs. A measure of relative efficiency of one design over another according to an optimality criterion aids in discriminating between the two designs for the "best" design. The D-, E-, A- and T-Optimal values of the general second order rotatable design in four dimensions constructed using balanced incomplete block designs when the number of replications (r) are less than three the number of times $(\lambda)$ pairs of treatments occur together in the design were found which may be used to determine the relative efficiency of the general design to the D-, E-, A- and T-Optimal designs.
\end{abstract}

Keywords: Optimal Designs, Rotatable Design, Balanced Incomplete Block Design and Optimal Values

\section{Introduction}

If the experimental region $(\mathrm{R})$ is either spherical or cuboidal, a standard response surface design such as central composite design (CCD), Box-Behnken design or their variation such as face-centred cube are applied since they are quite general and flexible [1]. But occasionally during experimentation these designs are not the obvious choice and Optimal designs are the alternative when the experimental region is irregular due to factor levels constraints or when the experimenter has prior knowledge about the process being studied which may suggest a non-standard model where some higher order terms or some interaction terms between factors may not be included in the model and the interest is to obtain an efficient design for fitting a reduced quadratic or higher order model or even when the process factors are categorical and finally an unusual sample size may be of importance due to cost or time considerations and a design of fewer trials is carried out [2]. Development of optimal designs is an outgrowth of work in the theory of optimal designs [3] and [4]. The efficiency of an experiment is greatly influenced by the adoption of an appropriate experimental design capable of representing the response surface design. Selecting an appropriate experimental design, is based on finding the best optimality criterion in which larger efficiency values imply a better design [5]. An optimal design is "best" with respect to some criterion which are single number criteria sometimes called alphabetical optimality criteria where each one intends to capture an aspect of the 'goodness' of a design. Response surface methodology (RSM) based on D-optimal design of experiments was employed to study the significance and interactive effect of methanol-to-oil $(\mathrm{M}: \mathrm{O})$ molar ratio, 
catalyst concentration, reaction time, and mixing rate on biodiesel yield [6]. Reference [7] found out that relationships between optimal design properties and changing sizes of designs by the addition of center points, between the Box-Benhken designs and the Box-Wilson designs was very strong. In this work we present the $D-, E-, A-$ and $T$-Optimal values of the general second order rotatable design in four dimensions constructed using balanced incomplete block designs when the number of replications (r) are less than three the number of times $(\lambda)$ pairs of treatments occur together in the design [8].

\section{Rotatability}

A design is rotatable if the prediction variance of response is constant at all points that are equidistant from the design center, which, by a proper coding of the control variables, can be chosen to be the point at the origin of the $\mathrm{k}$-dimensional co-ordinates system. Implying prediction variance is constant at all points that fall on the surface of a hypersphere centered at the origin i.e. if the design is rotatable, prediction variance remains unchanged under any rotation of the co-ordinate axes. If optimization of response is required on concentric hyperspheres, as is the case in ridge analysis, then it is important for the design to be rotatable [9].A class of three-level designs for estimating second-order response surfaces were proposed by [10]. Theses designs are rotatable or nearly so with a reduced number of experimental units by the $3^{n}$ designs. They are formed by combining $2^{n}$ designs with incomplete block designs.

\subsection{Rotatable Designs}

Given $k$ variates each at $s$ levels, a design formed with $N$ of the $s^{k}$ variates treatment combinations, can be written as $N \times K$ matrix, which we call the design matrix and denote it with $D_{N \times K}$ [9].

$$
D_{N \times K}=\left[\begin{array}{ccccc}
x_{11} & x_{21} & x_{31} & \ldots & x_{k 1} \\
x_{12} & x_{22} & x_{32} & \cdots & x_{k 2} \\
\cdots & \cdots & \cdots & \cdots & \cdots \\
\cdots & \cdots & \cdots & \cdots & \cdots \\
x_{1 N} & x_{2 N} & x_{3 N} & \cdots & x_{k N}
\end{array}\right]
$$

The treatment combinations are called points of the design and a design of the form described will be rotatable design of order $d$ if a response polynomial surface

$$
y_{u}=\beta_{0}+\sum_{i} \beta_{i} x_{i u}+\sum_{i \leq j} \beta_{i j} x_{i u} x_{j u}+\sum_{i \leq j \leq k} \beta_{i j k} x_{i u} x_{j u} x_{k u}+\cdots
$$

is obtained from the treatments, on the variables $x_{i}, i=$ $1,2, \ldots, k$ and with some suitable origin and scale, can be fitted so that the variance of the estimated response $\operatorname{var}[\hat{y}(x)]=\sigma^{2} x_{s}^{\prime}\left(X^{\prime} X\right)^{-1} x_{s}$ from any treatment is a function of the sum of squares of the levels of the factors in that treatment combination[11]. The variance of the estimate $y_{u}$ is only a function of the distance $\delta^{2}=\sum_{i=1}^{k} x_{i u}^{2}$ of the points $x_{1}, x_{2}, \ldots, x_{k}$ from the Centre of the design. Spherical variance of the estimation of the response surface is achieved if the design points satisfy the following conditions

$$
\sum_{u=1}^{N} x_{i u}=0, \sum_{u=1}^{N} x_{i u} x_{j u}=0, \sum_{u=1}^{N} x_{i u}^{2}=\sum_{u=1}^{N} x_{i u}^{2} x_{j u}^{2}=
$$

constant and $\sum_{u=1}^{N} x_{i u}^{4}=3 \sum_{u=1}^{N} x_{i u}^{2} x_{j u}^{2} \forall i=j$

11] and [8].

\subsection{Rotatable Design Using Balanced Incomplete Block Design (BIBD) with $r<3 \lambda$ )}

Reference [8] gave a method using properties of balanced incomplete block designs of obtaining second order rotatable deigns with any number of factors. For a four factor design, with number of replications $r$ of the BIBD being less than three the number of times $(\lambda)$ pairs of treatments occurred together in the design, the values for the coded levels of the design denoted by letters $a$ and $b$ were found to be \pm 1.137 and \pm 2.116 respectively as the coded levels of the factorial and the axial parts of the design.

\section{Parameter Estimation}

Given

$$
\begin{gathered}
y=\left[\begin{array}{c}
y_{1} \\
y_{2} \\
\vdots \\
y_{N}
\end{array}\right]_{N \times 1,} \quad \beta=\left[\begin{array}{c}
\beta_{1} \\
\beta_{2} \\
\vdots \\
\hat{a}_{n}
\end{array}\right]_{N \times 1,} \quad \epsilon=\left[\begin{array}{c}
\varepsilon_{1} \\
\varepsilon_{2} \\
\vdots \\
\varepsilon_{n}
\end{array}\right]_{N \times 1,} \\
X=\left[\begin{array}{ccccc}
1 & x_{11} & x_{12} & \cdots & x_{1 k} \\
1 & x_{21} & x_{22} & \cdots & x_{2 k} \\
\cdots & \cdots & \cdots & \cdots & \cdots \\
1 & x_{N 1} & x_{N 2} & \cdots & x_{N k}
\end{array}\right]_{N \times p}
\end{gathered}
$$

Where $y$ is an $(N \times 1)$ vector of observations at each run, $\beta$ is a $(N \times 1)$ vector of regression coefficients, $\epsilon$ is an $(N \times 1)$ vector of random errors and $X$ is an $(N \times p)$ matrix of levels of independent variables known as the model matrix with $p=k+1$

$$
y=X \beta+\epsilon
$$

We assume $\boldsymbol{\epsilon}$ is normally distributed with mean zero and $\operatorname{Cov}(\epsilon)=\sigma^{2} I[1]$. The estimate of the parameters is given by

$$
\hat{\beta}=\left(X^{\prime} X\right)^{-1} X^{\prime} y
$$

With their variances being

$$
\begin{gathered}
\operatorname{Var}(\hat{\beta})=\sigma^{2}\left(X^{\prime} X\right)^{-1} \\
\hat{y}_{u}=x_{u} \hat{\beta}
\end{gathered}
$$

The estimate of the response at $u^{t h}$ run is given as equation (8). Rotatability requires that the model have a reasonably consistent and stable variance throughout the region of interest $\mathrm{R}$. The variance of the predicted response at some point $x$ is

$$
V[\hat{y}(x)]=\sigma^{2} x^{\prime}\left(X^{\prime} X\right)^{-1} x
$$

Reference [11] suggested that a second order response surface should be rotatable meaning that the $V[y(x)]$ is the same at all points $x$ that are the same distance from the design center i.e. the variance of predicted response is constant on spheres. Since the 
aim of RSM is optimization and the location of the optimum point is unknown prior to running the experiment, it makes sense to use a design that provides equal precision of estimation in all the directions. The design problem therefore consists of selecting row vectors $X^{i \times p}, i=1,2, \ldots, N$ from the design space $X$ such that the design defined by these $N$ vectors is, in some sense, optimal. The moment matrix of the design is given as

$$
M=\frac{X^{\prime} X}{N}
$$

We assume $N$ is fixed. Solutions to this problem consists of developing some sensible criterion based on the above model and using it to obtain optimal designs [1].

\section{Optimal Designs and Optimality Criteria}

Optimal designs are constructed on the basis of a certain optimality criterion that pertains to the 'closeness' of the predicted response $\widehat{\boldsymbol{y}}(\boldsymbol{x})$ to the mean response, $\boldsymbol{\mu}(\boldsymbol{x})$ over a certain region of interest R [10]. Reference [4] developed useful computational procedures for finding optimal designs in regression problems of statistical inference. The approach is to specify the optimality criterion and then choose the design points from a grid of points spaced over the feasible design region [1]. An optimality criterion showed how good a design is. There are many optimality criteria, sometimes called alphabetical optimality criteria. These are single number criteria where each one intends to capture an aspect of the 'goodness' of a design and can be classified into either information-based criteria, distance-based criteria, compound design criteria and other criteria. Information-based criteria are related to the information matrix $X^{\prime} X$ of the design. This matrix is important because it is proportional to the inverse of the variance-covariance matrix for the least-squares estimates of the linear parameters of the model. These criteria can be divided into two classes according to the number of parameters used; the first class uses all parameters of the model and the second uses a sub-system of the parameters. In the first class, possible criteria to consider are, D-, A- and E- optimality criteria. Statistical models with several parameters have their mean of the parameters estimator as a vector making the variance of the parameters estimate a matrix whose inverse is called the "information matrix" The optimality properties of designs are determined by their moment matrices [12]. The class of $\phi_{p}$-criteria, that is T-, D-, A- and E- corresponding to parameter values $1,0,-1$ and $-\infty$ respectively are summarized in equation (11) as given in [12]. The amount of information inherent to $\mathrm{C}_{\mathrm{k}}(\mathrm{M}(\eta))$ is provided by $\phi_{p}$-criteria with $\mathrm{C}_{\mathrm{k}}(\mathrm{M}(\eta))$ $\in \mathrm{PD}(\mathrm{m})$, defined by:

$$
\begin{gathered}
\phi_{p}(C)=1 \\
\left\{\begin{array}{c}
\lambda_{\min }(C), \text { if } p=-\infty \\
\operatorname{det}(C)^{1 / s}, \text { if } p=0 \\
{\left[\frac{1}{s} \text { trace } C^{p}\right]^{p}, \text { if } p \neq 0, \pm \infty}
\end{array}\right.
\end{gathered}
$$

\subsection{D-optimality}

For information-based criteria, the most prominent of such criteria is the D-optimality criterion that maximizes $\left|X^{\prime} X\right|$ which amounts to the minimization of the size of the confidence region on the vector $\beta$ in the model [13].Reference [14] introduced Determinant criterion which emphasis on the quality of the parameter estimates. Later called, D-optimality by [4]. It's the most well studied problem seen in the literature by [3] and [15].Reference [12] considered the construction of D-optimal designs in a variety of examples and its popularity is due its simple computation, and the many available algorithms. D-Optimality is a parameter estimation criterion which aims at seeking designs which maximize the determinant of the information matrix. Let $\mathrm{C}$ be a parameter subsystem information matrix of $\mathrm{S}$ dimension, then D-optimality is given by

$$
\phi_{0}(C)=(\operatorname{det} C)^{1 / s}
$$

Maximization of the determinant of the information matrices is the same as minimizing the determinant of the dispersion matrices that is $(\operatorname{det} C)^{-1}=\operatorname{det}(C)^{-1}$. The focus of D-optimality is on estimation of model parameters through good attributes of the moment matrix, which is defined as equation (10) where $X^{\prime} X$ is the information matrix, and $N$, the total number of runs, which is used as a penalty for larger designs. D-optimality maximizes the determinant of the information matrix, i.e. Max $\left|X^{\prime} X\right|=\operatorname{Min}\left|\left(X^{\prime} X\right)^{-1}\right|$. Under the standard normality assumptions, $\left|X^{\prime} X\right|$ is inversely proportional to the square of the volume of the confidence region for the regression coefficients. Hence the larger the $\left|X^{\prime} X\right|$ better the estimation of the model parameters. A measure of relative efficiency of one design over another according to D-criterion is given by

$$
D_{\text {eff }}=\left[\frac{\left|\left(X_{1}^{\prime} X_{1}\right)^{-1}\right|}{\left|\left(X_{2}^{\prime} X_{2}\right)^{-1}\right|}\right]^{\frac{1}{s}} \text { Where } X_{1} \text { and } X_{2} \text { are the matrices }
$$
for the two designs and $s$ is the number of model parameters.

\subsection{E-Optimality}

Reference [16] introduced E-Optimality criterion in their work on relationships among several optimality criteria [17]. Computations of E-optimal designs for the full mean parameter vector and for many subsets in univariate polynomial regression models were determined by [18]. A method for computing E-optimal designs for a broad class of two parameter models was presented by [19]. The evaluation of the smallest eigen value of the information matrix $X^{\prime} X$ of a design is the same as minimizing the largest eigen value of the dispersion matrix $\left(X^{\prime} X\right)^{-1}[20]$. The procedure builds on finding the design which maximizes the minimum eigen value of $X^{\prime} X$ or equivalently, minimize the maximum eigen value of $\left(X^{\prime} X\right)^{-1}$. E-optimality aims at minimizing the maximum variance of all possible normalized linear combinations of parameter estimates.

$$
\operatorname{Max} \lambda_{\min }\left(X^{\prime} X\right)=\operatorname{Min} \lambda_{\max }\left(X^{\prime} X\right)^{-1}
$$

It is the minimization of the largest Eigen value of the dispersion matrix given by

$$
\frac{1}{\phi_{-\infty}\left(C_{k}(A)\right)}=\lambda_{\max }\left(C_{k}(A)^{-1}\right.
$$


The Eigen value criterion $\phi_{-\infty}$ is one of the four particular members of the one dimensional family of matrix means $\phi_{p}(p=-\infty)$ that submits itself to the principles that a reasonable criteria must meet as presented in [12].

$$
\phi_{-\infty}(C)=\lambda_{\min }(C)
$$

\subsection{A-Optimality}

Reference [21] introduced A-Optimality which involves the use of Fisher's information matrix as reported in [22]. It is an algebraic approach for constructing A-optimal design under generalized linear models as presented by [23]. This criteria aims at minimizing the sum of diagonal elements of the inverse of the moment matrix which is equivalent to minimizing the average variance of the parameter estimates given as Min trace $\left(X^{\prime} X\right)^{-1}$. If the coefficients matrix is partitioned into columns, $k=\left(C_{1}, C_{2}, \ldots, C_{k}\right)$ then the inverse $\frac{1}{\phi_{-1}}$ can be represented as

$$
\frac{1}{\phi_{-1}\left(C_{k}(A)\right)}=\frac{1}{s} \text { trace } C_{k}(A)^{-1}
$$

This corresponds to the average of the standardized variances of the optimal estimates of the scalar parameter systems $c_{1}^{\prime} \theta, \ldots, c_{s}{ }^{\prime} \theta$ formed from the columns of matrix $C$ as put forward by [12]. Average variance criterion is

$$
\phi_{-1}(C)=\left[\frac{1}{s} \operatorname{trace}^{-1}\right]^{-1}
$$

\subsection{T-Optimality}

T-optimal design is a plan where the optimality is obtained by discriminating between two or more models. Reference [24] introduced T-optimality design criterion in the context of optimal design theory. The T-criterion is given by

$$
\phi_{1}(C)=\frac{1}{S} \operatorname{trace} C
$$

\begin{tabular}{|c|c|c|c|c|c|c|c|c|c|c|c|c|c|c|}
\hline $\mathbf{X}_{0}$ & $X_{1}$ & $X_{2}$ & $\mathbf{X}_{3}$ & $X_{4}$ & $\mathbf{X}_{1} \mathbf{X}_{2}$ & $\mathbf{X}_{1} \mathbf{X}_{3}$ & $\mathrm{X}_{1} \mathrm{X}_{4}$ & $\mathbf{X}_{2} \mathbf{X}_{3}$ & $\mathrm{X}_{2} \mathrm{X}_{4}$ & $\mathbf{X}_{3} \mathbf{X}_{4}$ & $\mathrm{X}_{1}{ }^{2}$ & $\mathrm{X}_{2}{ }^{2}$ & $\mathrm{X}_{3}{ }^{2}$ & $X_{4}{ }^{2}$ \\
\hline 1 & -1.137 & 0 & -1.137 & -1.137 & 0 & 1.2928 & 1.2928 & 0 & 0 & 1.2928 & 1.2928 & 0 & 1.2928 & 1.2928 \\
\hline 1 & 1.137 & 0 & -1.137 & -1.137 & 0 & -1.2928 & -1.2928 & 0 & 0 & 1.2928 & 1.2928 & 0 & 1.2928 & 1.2928 \\
\hline 1 & -1.137 & 0 & 1.137 & -1.137 & 0 & -1.2928 & 1.2928 & 0 & 0 & -1.2928 & 1.2928 & 0 & 1.2928 & 1.2928 \\
\hline 1 & 1.137 & 0 & 1.137 & -1.137 & 0 & 1.2928 & -1.2928 & 0 & 0 & -1.2928 & 1.2928 & 0 & 1.2928 & 1.2928 \\
\hline 1 & -1.137 & 0 & -1.137 & 1.137 & 0 & 1.2928 & -1.2928 & 0 & 0 & -1.2928 & 1.2928 & 0 & 1.2928 & 1.2928 \\
\hline 1 & 1.137 & 0 & -1.137 & 1.137 & 0 & -1.2928 & 1.2928 & 0 & 0 & -1.2928 & 1.2928 & 0 & 1.2928 & 1.2928 \\
\hline 1 & -1.137 & 0 & 1.137 & 1.137 & 0 & -1.2928 & -1.2928 & 0 & 0 & 1.2928 & 1.2928 & 0 & 1.2928 & 1.2928 \\
\hline 1 & 1.137 & 0 & 1.137 & 1.137 & 0 & 1.2928 & 1.2928 & 0 & 0 & 1.2928 & 1.2928 & 0 & 1.2928 & 1.2928 \\
\hline 1 & -1.137 & -1.137 & 0 & -1.137 & 1.2928 & 0 & 1.2928 & 0 & 1.2928 & 0 & 1.2928 & 1.2928 & 0 & 1.2928 \\
\hline 1 & 1.137 & -1.137 & 0 & -1.137 & -1.2928 & 0 & -1.2928 & 0 & 1.2928 & 0 & 1.2928 & 1.2928 & 0 & 1.2928 \\
\hline 1 & -1.137 & 1.137 & 0 & -1.137 & -1.2928 & 0 & 1.2928 & 0 & -1.2928 & 0 & 1.2928 & 1.2928 & 0 & 1.2928 \\
\hline 1 & 1.137 & 1.137 & 0 & -1.137 & 1.2928 & 0 & -1.2928 & 0 & -1.2928 & 0 & 1.2928 & 1.2928 & 0 & 1.2928 \\
\hline 1 & -1.137 & -1.137 & 0 & 1.137 & 1.2928 & 0 & -1.2928 & 0 & -1.2928 & 0 & 1.2928 & 1.2928 & 0 & 1.2928 \\
\hline 1 & 1.137 & -1.137 & 0 & 1.137 & -1.2928 & 0 & 1.2928 & 0 & -1.2928 & 0 & 1.2928 & 1.2928 & 0 & 1.2928 \\
\hline 1 & -1.137 & 1.137 & 0 & 1.137 & -1.2928 & 0 & -1.2928 & 0 & 1.2928 & 0 & 1.2928 & 1.2928 & 0 & 1.2928 \\
\hline 1 & 1.137 & 1.137 & 0 & 1.137 & 1.2928 & 0 & 1.2928 & 0 & 1.2928 & 0 & 1.2928 & 1.2928 & 0 & 1.2928 \\
\hline 1 & -1.137 & -1.137 & -1.137 & 0 & 1.2928 & 1.2928 & 0 & 1.2928 & 0 & 0 & 1.2928 & 1.2928 & 1.2928 & 0 \\
\hline 1 & 1.137 & -1.137 & -1.137 & 0 & -1.2928 & -1.2928 & 0 & 1.2928 & 0 & 0 & 1.2928 & 1.2928 & 1.2928 & 0 \\
\hline 1 & -1.137 & 1.137 & -1.137 & 0 & -1.2928 & 1.2928 & 0 & -1.2928 & 0 & 0 & 1.2928 & 1.2928 & 1.2928 & 0 \\
\hline 1 & 1.137 & 1.137 & -1.137 & 0 & 1.2928 & -1.2928 & 0 & -1.2928 & 0 & 0 & 1.2928 & 1.2928 & 1.2928 & 0 \\
\hline 1 & -1.137 & -1.137 & 1.137 & 0 & 1.2928 & -1.2928 & 0 & -1.2928 & 0 & 0 & 1.2928 & 1.2928 & 1.2928 & 0 \\
\hline 1 & 1.137 & -1.137 & 1.137 & 0 & -1.2928 & 1.2928 & 0 & -1.2928 & 0 & 0 & 1.2928 & 1.2928 & 1.2928 & 0 \\
\hline 1 & -1.137 & 1.137 & 1.137 & 0 & -1.2928 & -1.2928 & 0 & 1.2928 & 0 & 0 & 1.2928 & 1.2928 & 1.2928 & 0 \\
\hline 1 & 1.137 & 1.137 & 1.137 & 0 & 1.2928 & 1.2928 & 0 & 1.2928 & 0 & 0 & 1.2928 & 1.2928 & 1.2928 & 0 \\
\hline 1 & 0 & -1.137 & -1.137 & -1.137 & 0 & 0 & 0 & 1.2928 & 1.2928 & 1.2928 & 0 & 1.2928 & 1.2928 & 1.2928 \\
\hline 1 & 0 & 1.137 & -1.137 & -1.137 & 0 & 0 & 0 & -1.2928 & -1.2928 & 1.2928 & 0 & 1.2928 & 1.2928 & 1.2928 \\
\hline 1 & 0 & -1.137 & 1.137 & -1.137 & 0 & 0 & 0 & -1.2928 & 1.2928 & -1.2928 & 0 & 1.2928 & 1.2928 & 1.2928 \\
\hline 1 & 0 & 1.137 & 1.137 & -1.137 & 0 & 0 & 0 & 1.2928 & -1.2928 & -1.2928 & 0 & 1.2928 & 1.2928 & 1.2928 \\
\hline 1 & 0 & -1.137 & -1.137 & 1.137 & 0 & 0 & 0 & 1.2928 & -1.2928 & -1.2928 & 0 & 1.2928 & 1.2928 & 1.2928 \\
\hline 1 & 0 & 1.137 & -1.137 & 1.137 & 0 & 0 & 0 & -1.2928 & 1.2928 & -1.2928 & 0 & 1.2928 & 1.2928 & 1.2928 \\
\hline 1 & 0 & -1.137 & 1.137 & 1.137 & 0 & 0 & 0 & -1.2928 & -1.2928 & 1.2928 & 0 & 1.2928 & 1.2928 & 1.2928 \\
\hline 1 & 0 & 1.137 & 1.137 & 1.137 & 0 & 0 & 0 & 1.2928 & 1.2928 & 1.2928 & 0 & 1.2928 & 1.2928 & 1.2928 \\
\hline 1 & 2.116 & 0 & 0 & 0 & 0 & 0 & 0 & 0 & 0 & 0 & 4.4775 & 0 & 0 & 0 \\
\hline 1 & -2.116 & 0 & 0 & 0 & 0 & 0 & 0 & 0 & 0 & 0 & 4.4775 & 0 & 0 & 0 \\
\hline 1 & 0 & 2.116 & 0 & 0 & 0 & 0 & 0 & 0 & 0 & 0 & 0 & 4.4775 & 0 & 0 \\
\hline 1 & 0 & -2.116 & 0 & 0 & 0 & 0 & 0 & 0 & 0 & 0 & 0 & 4.4775 & 0 & 0 \\
\hline 1 & 0 & 0 & 2.116 & 0 & 0 & 0 & 0 & 0 & 0 & 0 & 0 & 0 & 4.4775 & 0 \\
\hline 1 & 0 & 0 & -2.116 & 0 & 0 & 0 & 0 & 0 & 0 & 0 & 0 & 0 & 4.4775 & 0 \\
\hline 1 & 0 & 0 & 0 & 2.116 & 0 & 0 & 0 & 0 & 0 & 0 & 0 & 0 & 0 & 4.4775 \\
\hline 1 & 0 & 0 & 0 & -2.116 & 0 & 0 & 0 & 0 & 0 & 0 & 0 & 0 & 0 & 4.4775 \\
\hline
\end{tabular}

\section{Results}

Table 1. Full second order design matrix $X$. 
Table 2. Moment Matrix of the General Second Order Design.

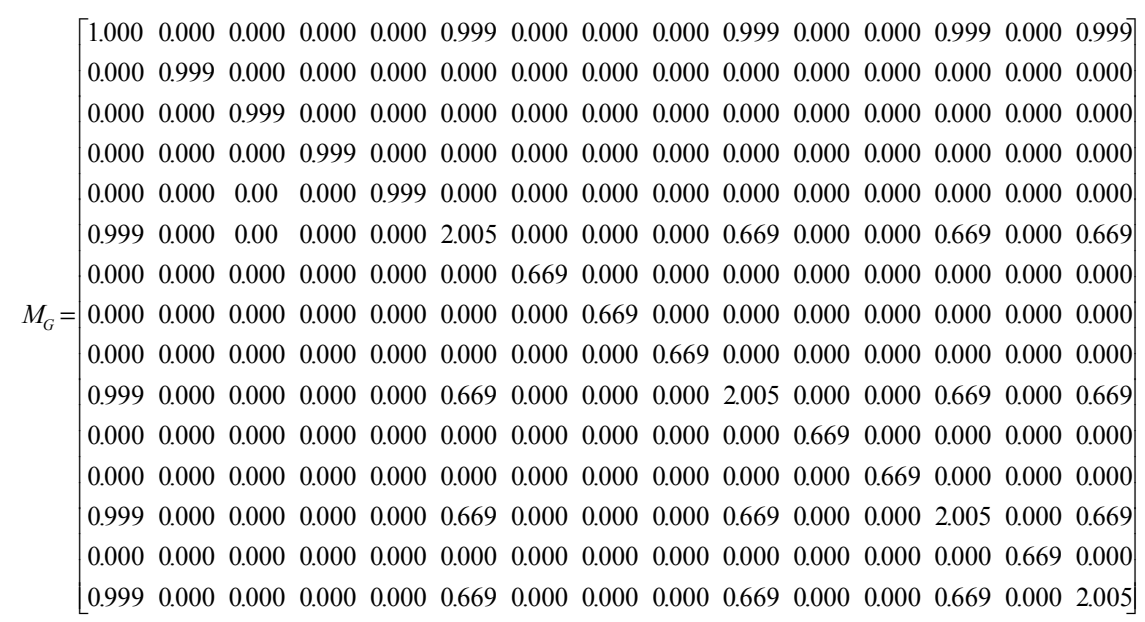

\subsection{D-Optimal Value}

Equation (12) with $C=M_{G}$ and $s=15$ (number of parameters in the model) was used.

$$
\phi_{0}\left(M_{G}\right)=\left(\operatorname{det} M_{G}\right)^{\frac{1}{15}}=0.6796529
$$

\subsection{E-Optimal}

Equation (15) with $C=M_{G}$ therefore the $E$-Optimal value

$$
\lambda_{\text {min }}\left(M_{G}\right)=0.002856958
$$

\subsection{A-Optimal Value}

Equation (17) with $C=M_{G}$ and $s=15$ gave

$$
\emptyset_{-1}(C)=\left(\frac{1}{15} \text { trace } M_{G}^{-1}\right)^{-1}=0.04104631
$$

\subsection{T-Optimal Value}

The trace criterion is useless if the regression vectors $x \in \chi$ have a constant squared length $\alpha$ say, then the moment matrix $M(\xi)$ of any design $\xi \in \Xi$ satisfies

$$
\text { Trace } M(\xi)=\text { trace } \int_{\chi} \chi \chi^{\prime} d \xi=\operatorname{trace} \int_{\chi} \chi^{\prime} \chi d \xi=c
$$

whence $\phi_{1}$ is a constant providing no distinction whatsoever [25] .For this design, the sum of the squares of the regression vectors $x \in \chi$ have different values as follows

$$
\begin{gathered}
\sum x_{0}^{2}=\sum x_{1}^{2}=\sum x_{2}^{2}=\sum x_{3}^{2}=\sum x_{4}^{2}=40, \\
\sum\left(x_{i}^{2}\right)^{2}=80.28822 \text { for } i=1,2,3, \& 4
\end{gathered}
$$

while

$$
\sum\left(x_{i} x_{j}\right)^{2}=26.76274
$$

Hence the trace will be of significance as optimality criteria. Equation (18) was applied

$$
\frac{1}{15} \operatorname{trace}\left(M_{G}\right)=1.135448
$$

\section{Conclusion}

$D-, A-, E-$ and $T-$ Optimal values of the general design were obtained as $0.6796529,0.04104631,0.002856958$, and 1.135448 respectively which may be used to determine the relative efficiency of the general design to the D-, E-, A - and T - Optimal designs. For example, D-optimal designs maximize the D-efficiency, which is a volume criterion on the generalized variance of the parameter estimates. The D-efficiency values are a function of the number of points in the design, the number of independent variables in the model and the maximum standard error for the prediction over the design points. The best design is the one with the highest D-efficiency which is also the case with the other optimality criterions in terms of their efficiencies.

\section{References}

[1] Montgomery D.C. (2005). Design and Analysis of Experiments. In (Fifth Edit) New York. John Wily \& Sons.

[2] Myers R.H. and Montgomery, D. C. (1995). Response Surace Methodology:Process and Product Optimization Using Designed Experiments. (Fifth Edit). John Wiley \$ sons.

[3] Kiefer, J. (1959). Optimum experimental designs. Journal of the Royal Statistical Society, B21, 272-319. https://doi.org/10.1007/s00184-011-0348-6

[4] Kiefer, J., \& Wolfowitz, J. (1959). Optimum designs in regression problems. Annals of Mathematical Statistics, 30, 271-294.

[5] Eze, F. C., \& O, L. N. (2018). Alphabetic Optimality Criteria for $2 K$ Central Composite Design Academic Journal of Applied Mathematical Sciences Alphabetic Optimality Criteria for $2 \mathrm{~K}$ Central Composite Design. September.

[6] El-Gendy, N. S., Ali, B. A., Abu Amr, S. S., Aziz, H. A., \& Mohamed, A. S. (2016). Application of D-optimal design and RSM to optimize the transesterification of waste cooking oil using natural and chemical heterogeneous catalyst. Energy Sources, Part A: Recovery, Utilization and Environmental Effects, 38 (13), 1852-1866. https://doi.org/10.1080/15567036.2014.967417 
[7] Iwundu, M. P. (2017). The Effects of Addition of n c Center Points on the Optimality of Box-Benhken and Box-Wilson Second-Order Designs. International Journal of Probability and Statistics, 6 (2), 20-32. https://doi.org/10.5923/j.ijps.20170602.02

[8] Das, M., \& Narasimham, V. (1962). Construction of rotatable desingns through balanced incomplete block desings. Annals of Mathematical Statistics, 33 (4).

[9] Khuri, A. I. (2017). Response Surface Methodology and Its Applications In Agricultural and Food Sciences. Biometrics \& Biostatistics International Journal, 5 (5). https://doi.org/10.15406/bbij.2017.05.00141.

[10] Box, G. E. P., \& Behnken, D. W. (1960). Some New Three Level Designs for the Study of Quantitative Variables. Technometrics, 2 (4), 455-475. https://doi.org/10.1080/00401706.1960.10489912.

[11] Box, G., \& Hunter, J. (1957). Multi-Factor Experimental Designs for Exploring Response Surfaces Author(s): G . E . P . Box and J.S. Hunter MUJLTI-FACTOR RESPONSE SURFACES '. The Annals of Mathematical Statistics, 28 (1), 195-241.

[12] Pukelsheim, F., \& Rosenberger, J. L. (1993). Experimental designs for model discrimination. Journal of the American Statistical Association, 88 (422), 642-649. https://doi.org/10.1080/01621459.1993.10476317

[13] Pazman, A. (1991). A Classification of N O NL IN E A R R E G R E S S I O N Models and. 8.

[14] Wald, A. (1943). On the Efficient Design of Statistical Investigations. The Annals of Mathematical Statistics, 14 (2), 134-140.

[15] Atkinson, A. and Donev, A. (1992). "Optimum experimental designs. Oxford University Press.

[16] Ehrenfeld, E. (1955). "On the efficiency of experimental design.” Annals of Mathematical Statistics, 26, 247-255.
[17] Rady, E. A., Abd El-Monsef, M. M. E., \& Seyam, M. M. (2009) Relationships among Several Optimality Criteria. Interstat Journals, 247, 1-11. http://interstat.statjournals.net/YEAR/2009/articles/0906001.p df

[18] Rissanen, J. (1983). Institute of Mathematical Statistics is collaborating with JSTOR to digitize, preserve, and extend access to The Annals of Statistics. ${ }^{\circledR}$ www.jstor.org. The Annals of Statistics, 11 (2), 416-431.

[19] Dette, H., \& Haines, L. M. (1994). E-optimal designs for linear and nonlinear models with two parameters. Biometrika, 81 (4), 739-754. https://doi.org/10.1093/biomet/81.4.739

[20] Pukelsheim, Fr. (2006). Optimal Design of Experiments. Society for industrial and applied mathematics.

[21] Smith, K. (1918). On the standard deviations of adjusted and interpolated values of an observed polynomial function and its constant and the guidance they give towards a proper choice of the distribution of observations. Biometrika, 12, 1-85.

[22] Chernoff, H. (1953). Locally optimal designs for estimating parameters. Annals of Statistics, 24, 586-602.

[23] Yang, M. (2008). A-optimal designs for generalized linear models with two parameters. Journal of Statistical Planning

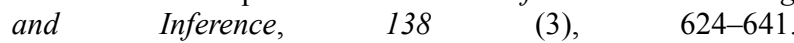
https://doi.org/10.1016/j.jspi.2006.12.007

[24] Atkinson, A. C., \& Fedorov, V. V. (1975). The design of experiments for discriminating between two rival models. Biometrika, $62 \quad$ (1), 57-70. https://doi.org/10.1093/biomet/62.1.57

[25] Pukelsheim, F., \& Studden, W. J. . E. (2007). Optimal Designs for Polynomial Regression. The Annals of Statistics, 21 (1), 402-415. https://doi.org/10.1214/aos/1176349033. 